\title{
Los cuentos infantiles y su rol en el desarrollo emocional en la primera infancia
}

\author{
[Children's storybooks and their role in \\ emotional development in early infancy]
}

\author{
Chamarrita Farkas (D)
}

Pontificia Universidad Católica de Chile

\begin{abstract}
Resumen
Este artículo aborda el desarrollo emocional en el período de la infancia temprana, con un énfasis en el desarrollo del reconocimiento y expresividad emocional, y su relevancia para el desarrollo integral de niñas y niños. Se examinan posibles dificultades en esta área y su relación con la incidencia de problemas de salud mental en preescolares chilenos, y su prevalencia. Se consideran algunos predictores del desarrollo emocional, considerando tanto la perspectiva madurativa, como la perspectiva social-contextual, y el proceso de socialización emocional. Se revisan dos intervenciones desarrolladas en Chile orientadas a promover tempranamente un desarrollo emocional sano: Las competencias parentales y los cuentos infantiles. Se discuten ventajas y limitaciones de dichas propuestas y se concluye que el uso de cuentos infantiles es la más recomendable considerando sus resultados, sus costos y su factibilidad de implementarse equitativamente en intervenciones que beneficien a niños y niñas.
\end{abstract}

Palabras clave: primera infancia, emociones, estrategia de promoción, competencias parentales, cuentos infantiles.

\begin{abstract}
In this article, we address the development of emotions in early infancy, emphasizing the emergence of emotional recognition and expressiveness and their relevance for children's overall development. We examine possible difficulties in this area and their relationship with the incidence and prevalence of mental health problems in Chilean preschool children. We consider some predictors of emotional development, including the maturational and social-contextual perspectives and the process of emotional socialization. We review two interventions aimed at early promotion of healthy emotional development in Chilean children: parental competencies and children's storybooks. We discuss these proposals in terms of their advantages and limitations and conclude that using children's storybooks is the most highly recommended considering the results, costs, and feasibility of equitable implementation as part of interventions that benefit all children.
\end{abstract}

Keywords: early infancy, emotions, promotion strategies, parental competencies, children's storybooks.

Contacto: La comunicación sobre este artículo debe ser enviada a Chamarrita Farkas, email chfarkas@uc.cl

Financiamiento: Esta investigación contó con financiamiento del Fondo Nacional de Desarrollo Científico y Tecnológico (FONDECYT 1180047) de la Agencia Nacional de Investigación y Desarrollo (ANID). 


\section{INTRODUCCIÓN}

Existe suficiente apoyo tanto de la literatura internacional como nacional sobre la relevancia de un adecuado desarrollo emocional en niñas y niños (por simplicidad, en adelante se usará la palabra niño para referirse a ambos sexos) desde temprana edad para su posterior desarrollo de competencias académicas, psicológicas y sociales en edades posteriores (Farkas et al., 2006; Palomera et al., 2012; Rhoades et al., 2011). No obstante, la prevalencia de problemas emocionales en los niños chilenos es bastante alta en comparación a otros países (Rescorla et al., 2011), lo cual hace extremadamente necesario un mayor conocimiento del desarrollo emocional de los niños durante los primeros cinco años de vida, así como posibles estrategias que promuevan un desarrollo adecuado en los niños, y que sean costo-efectivas. Así, este artículo tiene como objetivo revisar dos tipos de intervenciones que han demostrado ser exitosas en promover tempranamente un desarrollo emocional sano, desde una perspectiva tanto psicológica como educativa: las competencias parentales y los cuentos infantiles. Para ello, se parte revisando algunas nociones del desarrollo del reconocimiento y expresión emocionales, dos aspectos claves durante los primeros años. Se aborda su relevancia, y dentro de ello, la incidencia de dificultades en esta área que repercuten en la prevalencia de problemas de salud mental en los niños chilenos en este rango etario. Finalmente se revisan y discuten las dos estrategias sugeridas, considerando sus ventajas y limitaciones.

La tesis central de este trabajo es que la promoción de aspectos emocionales ha recibido una atención insuficiente en el desarrollo de políticas públicas orientadas a la primera infancia. En este contexto, el uso apropiado de cuentos infantiles para abordar temáticas emocionales es recomendable debido a que es una intervención que ha demostrado tener resultados exitosos, es factible de mantener en el tiempo, posibilita un acceso equitativo para todas las familias, y es de un menor costo en términos de recursos humanos y económicos.

\section{DESARROLLO EMOCIONAL EN LOS PRIMEROS AÑOS}

Desde muy temprano en su vida, los niños comienzan a adquirir habilidades emocionales y sociales que les permitirán adaptarse a las demandas y requerimientos del entorno social (Greenspan et al., 2001; Greenspan \& Shanker, 2004). Algunas de estas habilidades son el reconocer y entender las emociones propias y de otras personas, regular su expresión e identificar sus causas y consecuencias, y así usarlas para interactuar con otros de maneras positivas y adaptativas (Perner, 1994). Para ello, los niños deben atravesar ciertos hitos en el desarrollo de sus emociones. Un desarrollo adecuado llevará a un ajuste socioemocional sano. Conocer este desarrollo en los primeros años de vida es fundamental para distintos profesionales que trabajan en 
infancia temprana, para así promover oportunidades para un desarrollo en su máximo potencial, detectar oportunamente dificultades y generar intervenciones apropiadas.

Al nacer, los infantes no tienen conciencia de estos estados internos o emociones, pero ya desde los primeros meses comienzan a observarse ciertas conductas que dan cuenta del mundo emocional, como el contagio emocional y la sonrisa social (Perner, 1994). Además, antes del año los bebés son capaces de detectar cambios en los rostros de sus cuidadores y reaccionar a ellos, discriminando expresiones faciales y claves vocales y afectivas, y usándolas como referencia para interpretar su entorno (De Rosnay et al., 2006). A los dos años, hay una concepción mental de la emoción. Así, los niños pueden reconocer emociones; primero en términos de valencia (sentirse "bien" o "mal"), y luego como emociones discretas (feliz, triste, enojado entre los 24-36 meses, asustado y sorprendido a los 56 meses) (Widen \& Rusell, 2010). Gradualmente, la discriminación se especializa entre emociones de una misma gama emocional y se incorporan emociones más sociales y complejas (ej., vergüenza, culpa).

Respecto a la expresividad emocional, se ha planteado que desde el nacimiento el infante viene con un cierto set de emociones que se han denominado emociones básicas (i.e., alegría, tristeza, sorpresa, ira, miedo y asco). Estas emociones, de acuerdo con los planteamientos de Darwin, emergen tempranamente, tienen una base biológica y son universales a todos los seres humanos (Ekman, 1992). Inicialmente son expresiones globales de placer y displacer, y gradualmente se van volviendo más específicas y diferenciadas (Sroufe, 1996). Estas primeras expresiones emocionales son precursoras de las expresiones emocionales en los adultos. Cumplen funciones adaptativas y comunicacionales cruciales durante los primeros años, ayudan a los infantes a transmitir sus necesidades a otras personas, $\mathrm{y}$ tienen un rol fundamental en el desarrollo socioemocional temprano (Mesman et al., 2012).

\section{Influencia del contexto social en el desarrollo emocional}

La expresión y reconocimiento de emociones en el niño se va modificando no sólo por aspectos madurativos, sino por la influencia del contexto y del grupo social, dándose un proceso de socialización emocional. Lo que el niño desea o quiere entra en conflicto con los estándares familiares y sociales, por lo que éste tempranamente debe desarrollar una adecuada regulación emocional. Así, para que el niño pueda comportarse de maneras consideradas como socialmente apropiadas debe aprender a controlar y modular sus expresiones emocionales (Trommsdorff \& Cole, 2011). Esta socialización emocional varía en cada grupo social, y también es específica según aspectos como el sexo del niño o su nivel socioeconómico. Así, en la medida que el niño va creciendo, estas diferencias en la expresividad emocional se irán acentuando (Haapsamo et al., 2013; Muñoz \& Farkas, 2015).

Estudios con infantes chilenos y norteamericanos muestran diferencias en la expresión emocional entre ellos ya a los 12 meses, donde los niños chilenos exhibían una mayor autorregulación (Farkas \& Vallotton, 2016), y por tanto una menor intensidad en expresar placer y frustración (Muzard et al., 
2017). También se han encontrado diferencias en la intensidad y tipos de emoción que las madres demuestran según su nacionalidad y nivel socioeconómico (Muñoz et al., 2017). Lo anterior evidencia que desde temprana edad los niños están expuestos a distintos modelos sobre qué, cómo y cuánto demostrar de sus estados emocionales.

\section{Relevancia, dificultades, y prevalencia}

Estas habilidades emocionales tempranas son claves en el desarrollo de competencias psicológicas y sociales y para el logro del ajuste socioemocional (Palomera et al., 2012). Su adecuado desarrollo le permitirá al niño establecer relaciones positivas y de calidad con otros y comunicarse mejor con sus pares (Farkas et al., 2006), influirán positivamente en sus habilidades de aprendizaje y en su desempeño y éxito escolar (Rhoades et al., 2011) y se relacionarán con un desarrollo más positivo en la adultez (O'Connor et al., 2011).

Contrariamente, problemas en el desarrollo socioemocional de los niños conlleva al surgimiento de problemas emocionales y conductuales. Los problemas emocionales van en la línea de la ansiedad y la depresión, y en los primeros años pueden expresarse como problemas para separarse de figuras significativas, no querer ir al jardín o colegio, miedos y fobias, falta de energía y tristeza constante, por mencionar algunos. Si no son detectados y tratados a tiempo, se mantienen en el tiempo, mermando la salud mental durante la adolescencia y adultez (Bayer et al., 2006; Morgan et al., 2009). Problemas emocionales tempranos se asocian posteriormente con dificultades de aprendizaje, abandono escolar, victimización, y falta de proyectos vocacionales (Reijntjes et al., 2010). Estos problemas tienen una alta prevalencia (Egger \& Angold, 2006); estudios en distintos países muestran tasas de prevalencia entre $12 \%$ $29 \%$ (Belfer, 2008), y entre 25\%-48\% (Rescorla et al., 2007). Una mayor prevalencia de problemas de salud mental en la infancia se ha asociado con mayores niveles de inequidad social y económica (Reiss, 2013).

En Chile, la prevalencia de problemas emocionales en los niños es bastante alta en comparación a otros países (Rescorla et al., 2011). Estudios realizados hace 15 años o más, reportan tasas entre $10 \%$ y $15 \%$ de problemas emocionales en niños de la Región Metropolitana (Bralic et al., 1984; De la Barra et al., 2003; Delgado et al., 2006). Sin embargo, estos estudios fueron realizados hace bastante tiempo y con muestras muy pequeñas y por tanto no representativas a nivel nacional. En el 2010 se aplicó la primera versión de la "Encuesta Longitudinal de la Primera Infancia, ELPI”, la cual midió a 15,175 niños entre 7 y 58 meses y sus familias. Esta muestra es representativa de todas las regiones del país, incluyendo contextos urbanos y rurales. Un estudio realizado con los niños de 5 años de esta muestra reportó una prevalencia de 10.4\% de problemas emocionales en un nivel clínico, más un $12.4 \%$ de niños que se encontraban en un nivel de riesgo (Farkas et al., 2021).

Los problemas emocionales son bastante estables en la vida de una persona si no son tratados, y además con el tiempo se van intensificando (Bayer et al., 2006; Morgan et al., 2009). Ello plantea una carga sustancial para la salud pública, donde los problemas de salud mental tienen un alto impacto emocional y financiero para las 
personas afectadas, sus familias, y la sociedad en general (Minoletti \& Zaccaria, 2005). Para manejar estos problemas se han implementado dos planes nacionales de salud mental y psiquiatría en los años 1993 y 2000, y en el año 2011 se implementa el programa “Elige Vivir Sano", para lograr cumplir con los objetivos sanitarios de la década 2011-2020 (Minoletti \& Zaccaria, 2005; Valdés \& Errázuriz, 2012). No obstante, sigue existiendo un déficit financiero significativo en los fondos destinados a tratar estos problemas, en comparación a lo que se destina a nivel mundial. Al 2012 solo un $3.0 \%$ del presupuesto en salud se destinaba a salud mental, cuando el objetivo era lograr un 6\% (Valdés \& Errázuriz, 2012). Así, el financiamiento en salud mental en Chile sigue siendo una tarea pendiente, conllevando un fuerte detrimento de la calidad de vida de las personas y un substancial costo económico para el país (Errázuriz et al., 2015). Esta situación es aún más severa al revisar la salud mental de los niños, quienes son los que tienen las mayores cifras de pobreza y un menor acceso a una atención especializada (Ministerio de Salud, 2017).

Por ello son de gran relevancia programas de promoción temprana de un adecuado desarrollo socioemocional y la detección e intervención oportunas en los problemas emocionales infantiles. En Chile, y de manera coherente con estudios internacionales, aspectos como vivir en zonas rurales, el desempleo materno y bajos niveles educativos son factores de riesgo para los problemas emocionales de los niños (Farkas et al., 2021). Pero estos son factores contextuales muy difíciles de modificar a corto plazo. Ello plantea la pregunta sobre qué otros aspectos podrían promover un adecuado desarrollo emocional en niños pequeños, con intervenciones que sean costo-efectivas, y que a la vez sean factibles de implementarse con resultados a corto plazo. Estos aspectos se revisan en la siguiente sección.

\section{PROMOVIENDO UN ADECUADO DESARROLLO EMOCIONAL EN LA INFANCIA}

Otros factores de riesgo que se han estudiado para los problemas emocionales abordan aspectos individuales de los niños, como por ejemplo sexo, temperamento, y estilo de apego; aspectos de los padres, como su personalidad y antecedentes de salud mental; y aspectos de la interacción, como por ejemplo estilos de disciplina y crianza, el tipo de actividades a través de las cuales interactúan (e.g., rutinas, juego, cuentos), y las competencias parentales que se ponen en juego en dichas interacciones (ver por ejemplo Farkas \& Vallotton, 2016; Marrone, 2002; Morgan et al., 2009; Ramos et al., 2020). Ello ha generado variadas intervenciones orientadas a los niños, a los padres o a ambos, con intervenciones individuales o grupales, clínicas o psicoeducativas, y usando distintas herramientas como el juego, materiales como títeres, técnicas gráficas y pictóricas, y talleres para padres, por mencionar algunas. Todas ellas cuentan con mayor o menor evidencia empírica que las sustentan, pero no necesariamente son factibles de llevarse a cabo si se piensa en términos de costo-beneficio, posibilidad de implementarse a nivel nacional y con un acceso equitativo para todas las familias. 
Así, en esta sección se revisan los trabajos en dos tipos de intervención que predicen un desarrollo adecuado de las emociones en la infancia temprana, y que podrían implementarse a escala nacional: las competencias parentales, y el uso de los cuentos infantiles.

\section{Competencias parentales}

La familia es el primer escenario donde se inicia el desarrollo emocional del niño, por lo que los padres tienen un rol primordial en promover un desarrollo adecuado en su hijo, a través del ejercicio de sus competencias parentales. Estas competencias aluden al conjunto de habilidades que permiten a los padres afrontar las necesidades evolutivas y educativas de sus hijos, de manera flexible y adaptativa, y con los estándares considerados como aceptables por la sociedad (Rodrigo et al., 2008). Las competencias parentales más estudiadas en el último tiempo son la sensibilidad y la mentalización. La sensibilidad se refiere a la capacidad de los padres de percibir e interpretar apropiadamente las señales del niño, y de responder a ellas de manera oportuna y adecuada (Ainsworth et al., 1978; Marrone, 2002). Generalmente esta competencia ha sido estudiada en las madres, y la sensibilidad materna ha demostrado ser un predictor significativo de las habilidades sociales y logros académicos de los niños en sus primeras etapas, e incluso hasta la adolescencia (Raby et al., 2015). Estudios con padres, aunque más escasos, muestran que el tipo de relación e involucramiento entre padres e hijos influye en su desarrollo cognitivo, del lenguaje, emocional y social (Cabrera et al., 2007). Estudios con niños chilenos relacionan la sensibilidad materna con el desarrollo socioemocional de los niños a los 12 meses, y ponen el acento en el rol de esta competencia durante el primer año del niño (Farkas et al., 2017; Ramos et al., 2020).

La mentalización parental se refiere a la habilidad de los padres de percibir a sus hijos como seres con mente, y capaces de tener por tanto conductas intencionales (Meins et al., 1998). Ello influye el lenguaje que los padres utilizan al interactuar con sus hijos -denominado como lenguaje mentalizantey el cual se caracteriza por incluir referencias a estados mentales tales como deseos, pensamientos y emociones (Ruffman et al., 2012). Esta competencia ha sido relacionada con el desarrollo emocional del niño, con su comprensión de las emociones y con su desarrollo temprano de competencias comunicativas (Slaughter \& Peterson, 2009; Taumoepeau \& Ruffman, 2008). En niños chilenos se ha observado que un mayor uso de términos emocionales por parte de las madres durante el juego predecía el desarrollo socioemocional de los niños un año y medio después (Farkas et al., 2018).

Investigaciones han demostrado que intervenciones preventivas, tempranas y acotadas en sensibilidad parental, son efectivas (para más detalle ver Bakermans-Kranenburg et al., 2003). Asimismo, aunque menos estudiado, intervenciones grupales en personal educativo también han demostrado ser exitosas (Santelices et al., 2017; Werner et al., 2016). Se han desarrollado además intervenciones en mentalización tanto en padres como en educadores, de tipo individual y grupal, y con intervenciones psicoeducativas, terapéuticas, y en visitas domiciliarias (ver Santelices et al., 2016), que han demostrado ser efectivas (Adkins et al., 2018; Asen \& Fonagy, 2012). 
Este tipo de intervenciones presentan una serie de ventajas, siendo las más relevantes el que han demostrado ser altamente efectivas en distintos tipos de contextos, requerir tiempos relativamente breves de tiempo, y tener un impacto positivo en diferentes aspectos de los niños. No obstante, también contemplan algunas limitaciones. En primer lugar, requieren de capacitación para poder ser implementadas, capacitación que puede requerir un menor o mayor tiempo según el tipo de intervención a realizar, pero que conlleva una serie de costos, considerando el entrenamiento de los profesionales, y la necesidad de formación continua y supervisión de estas habilidades. A ello se suma la necesidad constante de formar nuevos profesionales, considerando las altas tasas de rotación en Chile del personal educativo y de la salud, especialmente en los sistemas públicos (Carrasco et al., 2017; Universidad de Chile et al., 2014).

Otra limitación refiere a la implementación de estas intervenciones de manera equitativa a nivel nacional. Como ya se mencionó, el presupuesto destinado a salud mental es insuficiente (Errázuriz et al., 2015). Ello influye en una menor oferta de atención, una mayor demora en los tiempos para acceder a los especialistas, tiempos de atención más breves, y un énfasis en los problemas de mayor complejidad versus el desarrollo de estrategias preventivas. Además, el acceso a esta atención es inequitativo según geografía, ruralidad, culturas originarias y por tipo de previsión (Universidad de Chile et al., 2014). Lo anterior lleva a plantearse la necesidad de pensar en otro tipo de estrategias.

\section{Cuentos infantiles como herramienta de prevención y promoción}

Los cuentos infantiles tienen una serie de beneficios para los niños ya que son entretenidos, son una fuente de información, ayudan a desarrollar su sentido estético, y los acercan tanto a mundos reales como imaginarios, fomentando su imaginación y creatividad (Arévalo, 2011; Montoya, 2004). Apoyan el desarrollo del pensamiento y del lenguaje infantil, la adquisición de un vocabulario más rico y diverso, y una mejor comprensión lectora (Hoyos Londoño, 2015; Macías, 2010). Aportan al desarrollo de la personalidad del niño, promoviendo una autoestima más positiva y mejores habilidades de autorregulación (Gonçalves, 2015).

Los cuentos ofrecen un contexto privilegiado donde el niño puede comprender su mundo interno. Las narrativas presentes dependen de estados psicológicos para conectar los eventos y así dar coherencia a la historia, lo cual incentiva un mayor uso de un lenguaje mentalizante, incluyendo términos emocionales (Farkas, 2019; Farkas et al., 2018). Durante la lectura compartida de cuentos aparecen más frecuentemente palabras relacionadas con el mundo interno, en comparación a interacciones de juego adulto-niño (Dyer et al., 2000; Farkas et al., 2018). Además, el texto de los cuentos para niños de 3 a 5 años muestra un abundante uso de términos emocionales (en frecuencia y en diversidad del vocabulario) (Farkas, Santelices et al., 2020). Entre ellos, las emociones más predominantes son felicidad, pena, miedo y rabia (Farkas, Gerber et al., 2020), lo cual es coherente con el desarrollo de las primeras etiquetas emocionales en los niños (Widen \& Rusell, 2010). 
Existe amplia evidencia de que la lectura compartida de cuentos infantiles es beneficiosa para el desarrollo del lenguaje. Pero también se ha comprobado su aporte al desarrollo de las habilidades socioemocionales en los niños (O'Farrelly et al., 2018), apoyando el desarrollo de la imaginación, juego social, interacciones sociales y conductas prosociales en preescolares (Kohm et al., 2016), y promoviendo una sensibilidad multicultural (Kim et al., 2006).

Lo anterior plantea el tema del tipo de cuentos a los cuales los niños están expuestos. Se ha visto que cuando las madres eran capaces de elegir cuentos adecuados para el desarrollo del niño, ello predecía la empatía y ajuste socioemocional infantil (Aram \& Aviram, 2009). Además, hay diferencias en las emociones más frecuentes en los cuentos disponibles en distintas culturas (Farkas, Gerber et al., 2020; Tsai et al., 2007). Se ha planteado que esas diferencias son coherentes con la socialización de emociones propias a cada cultura, y la exposición diferencial de los niños a ciertos cuentos moldea su expresividad emocional (Tsai et al., 2007).

\section{CONCLUSIONES}

Suficiente literatura internacional y nacional apoya el que un adecuado desarrollo emocional en los niños desde temprana edad es de gran relevancia para el posterior desarrollo socioemocional y ajuste social, académico y laboral de las personas. A su vez, promover tempranamente un desarrollo adecuado de los aspectos emocionales reduce a futuro el surgimiento de problemas emocionales, siendo beneficioso para la salud mental de los niños y con un costo menor a aquellas intervenciones que se requieren en edades posteriores.

Esto plantea un desafío para el desarrollo de políticas públicas que consideren la promoción del desarrollo emocional en los primeros cinco años de vida. Algunos esfuerzos en esta línea han comenzado a realizarse; por ejemplo, la creación a partir del 2007 del Sistema de Protección Integral a la Infancia (Programa Chile Crece Contigo). Pero, aunque este programa está siendo beneficioso en una serie de aspectos de los niños y sus familias, los aspectos emocionales han recibido una atención insuficiente (ver por ejemplo Castillo et al., 2018).

Previamente fueron sugeridas dos vías para promover aspectos emocionales de los niños: el apoyo de competencias parentales como sensibilidad y mentalización, y el uso de cuentos infantiles. La promoción de competencias parentales saludables, $\mathrm{o}$ la intervención en aquellos casos donde se presentan dificultades, ha demostrado ser eficaz a través de distintas estrategias; con intervenciones individuales y grupales, y con aproximaciones psicoterapéuticas y psicoeducativas. Sin embargo, esta línea presenta varias limitaciones ya discutidas previamente, y que se sintetizan en un mayor costo en recursos humanos y económicos, y dificultades para implementarlas de una manera que asegure su mantención en el tiempo y un acceso equitativo de todas las familias, independientemente de donde vivan. El uso de cuentos infantiles apropiados para abordar temáticas emocionales podría subsanar en parte 
estas limitaciones. Los cuentos seleccionados para apoyar el desarrollo emocional de los niños plantean una inversión inicial, pero luego es más fácil mantenerlos en el tiempo, y su costo en recursos humanos y económicos es menor a la implementación de intervenciones de apoyo a las competencias parentales por parte de profesionales. La forma elegida para difundirlos facilitaría su acceso a familias de distintas zonas del país, especialmente aquellas que viven en zonas rurales y con mayor desprotección. Además, los cuentos son un recurso que perdura en el tiempo, pudiendo ser utilizados por distintas familias. No obstante, también plantean varios desafíos. El primero y más complejo es la baja tasa con que los padres leen cuentos infantiles a sus hijos. En un estudio realizado con niños de 5 años de la región metropolitana, solo un $54.7 \%$ de los padres declaró haber leído al menos una vez con su hijo durante la última semana, y ello además se veía relacionado directamente con el nivel educacional de los padres; un $27.5 \%$ de padres que solo habían llegado a la enseñanza media reportó tener 10 o más libros infantiles en el hogar (Strasser \& Lissi, 2009). Ello plantea el desafío de cómo lograr que las familias lean los cuentos, especialmente aquellas de niveles socioeconómicos y educacionales más bajos, que son donde hay más necesidad de apoyar el desarrollo emocional de los niños. Desarrollar hábitos de lectura en la edad adulta es extremadamente difícil, pero es durante los primeros años que estas habilidades deben potenciarse.

Un segundo desafío es la selección de cuentos infantiles que sean más apropiados para potenciar el desarrollo emocional de los niños, que tomen en consideración tanto aspectos propios a su maduración como a las temáticas más relevantes a abordar, siendo a la vez historias atractivas que motiven su lectura. Ello, ya que hay bastante heterogeneidad entre los cuentos respecto a la cantidad de páginas, temática que abordan, cantidad y tipo de vocabulario utilizado, frecuencia de términos emocionales y oportunidades potenciales para hablar de las emociones. También es relevante considerar que, por su edad, los niños pequeños no leen y por tanto especial atención debe prestarse a las imágenes que aparecen en los cuentos, con personajes que sean expresivos y coherentes en su emocionalidad, y que les permitan a los niños reconocerlos e identificarse. Una posible forma de abordar este desafío sería conformar un panel multidisciplinario de expertos que revise y sugiera una lista de cuentos para ser leídos por las familias y en centros educativos y de protección y cuidado de niños, e instrucciones sobre su uso. Un tercer y último desafío es cómo implementar el acceso a los cuentos por parte de las familias. Los libros en general en Chile son caros, la gran mayoría de los cuentos de calidad son importados lo cual aumenta sus costos, hay poca oferta y poca diversidad en formatos de menor costo y más accesibles, como por ejemplo libros digitales. Aunque en el último tiempo han comenzado a aparecer fundaciones y organizaciones que buscan apoyar la lectura de cuentos en la infancia, el desarrollo de una política pública que invierta fondos en este aspecto sería de gran relevancia, considerando esto como una inversión a mediano y largo plazo en el desarrollo de los niños chilenos, no solo en promover su desarrollo emocional y social, sino de su lenguaje, su comprensión lectora y su creatividad. 


\section{REFERENCIAS}

Adkins, T., Luyten, P., \& Fonagy, P. (2018). Development and preliminary evaluation of family minds: A mentalization-based psychoeducation program for foster parents. Journal of Child and Family Studies, 27(8), 2519-2532. https://doi.org/10.1007/s10826-018-1080-x

Ainsworth, M. D., Blehar, M. C., \& Waters, E. (1978). Patterns of attachment. Lawrence Erlbaum Associates.

Aram, D., \& Aviram, S. (2009). Mothers' storybook reading and kindergartners' socioemotional and literacy development. Reading Psychology, $\quad 30(2), \quad$ 175-194. https://doi.org/10.1080/02702710802275348

Arévalo, J. P. (2011). La literatura infantil, un mundo por descubrir. Editorial Visión Libros.

Asen, E., \& Fonagy, P. (2012). Mentalizationbased therapeutic interventions for families. Journal of Family Therapy, 34(4), 347-370. https://doi.org/10.1111/j.1467-6427.2011.00552.x

Bakermans-Kranenburg, M. J., Van Ijzendoorn, M. H., \& Juffer, F. (2003). Less is more: Meta-analyses of sensitivity and attachment interventions in early childhood. Psychological Bulletin, 129(2), 195-215. https://doi.org/10.1037/0033-2909.129.2.195

Bayer, J. K., Sanson, A. V., \& Hemphill, S. A. (2006). Parent influences on early childhood internalizing difficulties. Journal of Applied Developmental Psychology, 276), 542-559. https://doi.org/10.1016/j.appdev.2006.08.002
Belfer, M. L. (2008). Child and adolescent mental disorders: The magnitude of the problem across the globe. Journal of Child Psychology and Psychiatry, 49(3), 226-236.

https://doi.org/10.1111/j.1469-7610.2007.01855.x

Bralic, S., Seguel, X., \& Montenegro, H. (1984). Prevalencia de trastornos psíquicos en la población escolar de Santiago. CEDEP UNICEF.

Cabrera, N. J., Shannon, J. D., \& TamisLeMonda, C. (2007). Fathers' influence on their children's cognitive and emotional development: From toddlers to pre-K. Applied Development Science,11(4), 208-213. https://doi.org/10.1080/10888690701762100

Carrasco, D., Godoy, M. I., \& Rivera, M. (2017). Rotación de profesores en Chile: Quiénes son y cuál es el contexto de quienes dejan su primer trabajo. MidEvidencias, (11), 1-7. https://www.mideuc.cl/wp-content/uploads/ 2016/MidEvidencias-N11.pdf

Castillo, C., Quezada, S., Valenzuela, P., \& Silva, V. (2018). A diez años de Chile Crece Contigo. Claves y aprendizajes para la construcción de sistemas comprehensivos de apoyo al desarrollo infantil. Ministerio de Desarrollo Social y Familia; Banco Mundial.

Gonçalves, R. (2015). Conflictos psíquicos en la infancia y cuentos de hadas: Los cuentos infantiles como dispositivo de intervención en la práctica clínica. Subjetividad $y$ Procesos Cognitivos, 19(1), 131-148. https://redalyc.org/pdf/3396/339641097008.pdf 

el desarrollo emocional en la primera infancia. Revista de Sociología, 36(1), 68-82. https://doi.org/10.5354/0719-529X.2021.64427

De la Barra, F., Toledo, V., \& Rodríguez, J. (2003). Estudio de salud mental en dos cohortes de niños escolares de Santiago Occidente III: Predictores tempranos de problemas conductuales y cognitivos. Revista Chilena de Neuropsiquiatría, 41(1), 65-76. https://doi.org/10.4067/S071792272003000100008

De Rosnay, M., Cooper, P. J., Tsigaras, N., \& Murray, L. (2006). Transmission of social anxiety from mother to infant: An experimental study using a social referencing paradigm. Behaviour Research and Therapy, 44(8), 1165-1175. https://doi.org/10.1016/j.brat.2005.09.003

Delgado, I., Zuñiga, V., \& Jadue, L. (2006). Consultoria "Estudio comparativo de escolares que participaron del programa habilidades para la vida y SIMCE $4^{\circ}$ básico 2005"[Resumen Ejecutivo]. Junaeb; Gobierno de Chile.

Dyer, J. R., Shatz, M., \& Wellman, H. M. (2000). Young children's storybooks as a source of mental state information. Cognitive Development, 15(1), 17-37. https://doi.org/10.1016/S0885-2014(00)00017-4

Egger, H. L., \& Angold, A. (2006). Common emotional and behavioral disorders in preschool children: Presentation, nosology, and epidemiology. Journal of Child Psychology and Psychiatry, 473-4), 313337. http://doi.org/10.1111/j.1469-7610.2006.01618.x Ekman, P. (1992). Are there basic emotions? Psychological Review, 99(3), 550-553 https://doi.org/10.1037/0033-295X.99.3.550

Errázuriz, P., Valdés, C., Vöhringer, P. A., \& Calvo, E. (2015). Financiamiento de la salud mental en Chile: Una deuda pendiente. Revista Médica de Chile, 143(9), 1179-1186. http://doi.org/10.4067/S0034-98872015000900011
Farkas, C. (2019). Hablemos de emociones, sensaciones y pensamientos en los cuentos infantiles. Beneficios para los niños y niñas preescolares [Sesión de conferencia]. Primer Congreso Internacional de Lectura y Primera Infancia, IBBY Chile y Fundación Entrelíneas, Santiago, Chile.

Farkas, C., del Real, M. T., Strasser, K., Álvarez, C., Santelices, M. P., \& Sieverson, C. (2018). Maternal mental state language during storytelling versus free-play contexts and its relation to child language and socioemotional outcomes at 12 and 30 months of age. Cognitive Development, 47, 181-197. https://doi.org/10.1016/j.cogdev.2018.06.009

Farkas, C., Gerber, D., Mata, C., \& Santelices, M. P. (2020). Are children from different countries exposed to diverse emotions in storybooks? Comparative study between Chile and the US. Social Development, 29(4),11341154. https://doi.org/10.1111/sode. 12443

Farkas, C., Girard, L.-C., \& MacBeth, A. (2021). Predictors of emotional problems in 5-yearold children: An international comparison between two cohorts in Chile and Scotland. Current Psychology. Publicación anticipada en línea. https://doi.org/10.1007/s12144-020-01336-5

Farkas, C., Grothusen, S., Muñoz, M. J., \& von Freeden, P. (2006). Revisión de las habilidades socio-cognitivas en la infancia temprana. Summa Psicológica, 3(1), 31-42. https://doi.org/10.18774/448x.2006.3.31

Farkas, C., Santelices, M. P., Vallotton, C. D., Brophy-Herb, H. E., Iglesias, M., Sieverson, C., Cuellar, M. P., \& Álvarez, C. (2020). Children's storybooks as a source of mental 

el desarrollo emocional en la primera infancia. Revista de Sociología, 36(1), 68-82. https://doi.org/10.5354/0719-529X.2021.64427

state references: Comparison between books from Chile, Colombia, Scotland and USA. Cognitive Development, 53, Artículo 100845 https://doi.org/10.1016/j.cogdev.2019.100845 Farkas, C., \& Vallotton, C. (2016). Differences in infant temperament in Chile and the US. Infant Behavior \& Development, 44, 208-218. https://doi.org/10.1016/j.infbeh.2016.07.005

Farkas, C., Vallotton, C. D., Strasser, K., Santelices, M. P., \& Himmel, E. (2017). Socioemotional skills between 12 and 30 months of age on Chilean children: When do the competences of adults matter? Infant Behavior \& Development, 49, 192-203. https://doi.org/10.1016/j.infbeh.2017.09.010

Greenspan, S. I., \& Shanker, S. (2004). The first idea: How symbols, language, and intelligence evolved from our primate ancestors to modern humans. Perseus Books.

Greenspan, S. I., DeGangi, G. A., \& Wieder, S. (2001). The functional emotional assessment scale (FEAS) for infancy and early childhood: Clinical and research applications. Interdisciplinary Council of Developmental and Learning Disorders.

Haapsamo, H., Kuusikko-Gauffin, S., Ebeling, H., Larinen, K., Penninkilampi-kerola, V., Soini, H., \& Moilanem, I. (2013). Communication development and characteristics of influencing factors: A followup study from 8 to 36 months. Early Child Development and Care, 183(2),321-334. https://doi.org/10.1080/03004430.2012.674523

Hoyos Londoño, M. C. (2015). La literatura infantil y sus beneficios en el desarrollo del pensamiento y del lenguaje. Revista Katharsis, (19), 73-98. https://doi.org/ 10.25057/25005731.490

Kim, B. S., Green, J. L. G., \& Klein, E. F. (2006). Using storybooks to promote multicultural sensitivity in elementary school children. Journal of Multicultural Counseling and Development, 34(4), 223-234. https://doi.org /10.1002/j.2161-1912.2006.tb00041.x

Kohm, K. E., Holmes, R. M., Romeo, L., \& Koolidge, L. (2016). The connection between shared storybook readings, children's imagination, social interactions, affect, prosocial behavior, and social play. International Journal of Play, 5(2), 128-140. https://doi.org/10.1080/21594937.2016.1203895

Macías, M. C. M. (2010). Los beneficios de la literatura infantil. Revista Digital para Profesionales de la Enseñanza, (8), 1-6. https://www.feandalucia.ccoo.es/andalucia/d ocu/p5sd7247.pdf

Marrone, M. (2002). La teoría del apego: Un enfoque actual. Psimática.

Meins, E., Fernyhough, C., \& Russell, J. (1998). Security of attachment as a predictor of symbolic and mentalising abilities: A longitudinal study. Social Development, 71), 1-24. https://doi.org/10.1111/14679507.00047.

Mesman, J., Oster, H., \& Camras, L. (2012). Parental sensitivity to infant distress: What do discrete negative emotions have to do with it? Attachment \& Human Development, $\quad$ 14(4), 337-348. https://doi.org/10.1080/14616734.2012.6916649 

el desarrollo emocional en la primera infancia. Revista de Sociología, 36(1), 68-82. https://doi.org/10.5354/0719-529X.2021.64427

Ministerio de Salud (2017). Plan nacional de salud mental 2017-2025. Gobierno de Chile. https://www.minsal.cl/wp-content/uploads/2017/ 12/PDF-PLAN-NACIONAL-SALUD-MENTAL2017-A-2025.-7-dic-2017.pdf

Minoletti, A., \& Zaccaria, A. (2005). Plan nacional de salud mental en Chile: 10 años de experiencia. Revista Panamericana de Salud Pública, 18(4-5), 346-358. http://www.scielosp.org/article/rpsp/2005.v1 8n4-5/346-358/es/

Montoya, V. (2004). El poder de la fantasía y la literatura infantil. Ediciones del Sur. http://sincronia.cucsh.udg.mx/litinfant.htm

Morgan, P. L., Farkas, G., \& Wu, Q. (2009). Kindergarten predictors of recurring externalizing and internalizing psychopathology in the third and fifth grades. Journal of Emotional and Behavioral Disorders, 17(2), 67-79. https://doi.org/10.1177/1063426608324724

Muñoz, P., \& Farkas, C. (2015). Reflexiones sobre la relación entre expresión emocional y cultura. Revista Humanitas, 12(12), 140-155. http://rhumanitas.ucatolica.ac.cr/Articulos/Index/25

Muñoz, P., Farkas, C., \& Vallotton, C.D. (2017). Gestural representation of emotions: A comparative study on Chilean and American women. Journal of Child and Family Studies, 26(8), 2166-2174. https://doi.org/10.1007/s10826-017-0724-6

Muzard, A., Kwon, A. Y., Espinosa, N., Vallotton, C. D., \& Farkas, C. (2017). Infants' emotional expression: Differences in the expression of pleasure and discomfort between infants from Chile and the United States. Infant and Child Development, 26(6), 1-12.

https://doi.org/10.1002/icd.2033
O'Connor, M., Sanson, A., Hawkins, M. T., Letcher, P., Toumbourou, J. W., Smart, D., Vasallo, S., \& Olsson, C. A. (2011). Predictors of positive development in emerging adulthood. Journal of Youth and Adolescence, 40(7), 860-874. https://doi.org/10.1007/s10964-010-9593-7

O'Farrelly, C., Doyle, O., Victory, G., \& Palamaro-Munsell, E. (2018). Shared reading in infancy and later development: Evidence from an early intervention. Journal of Applied Developmental Psychology, 54, 69-83. https://doi.org/10.1016/j.appdev.2017.12.001

Palomera, R., Salguero, J. M., \& Ruiz-Aranda, D. (2012). La percepción emocional como predictor estable del ajuste psicosocial en la adolescencia. Behavioral Psychology / Psicología Conductual. Revista Internacional de Psicología Clínica y de la Salud, 20(1), 43-58. https://www.behavioralpsycho.com/producto /la-percepcion-emocional-como-predictorestable-del-ajuste-psicosocial-en-la-adolescencial

Perner, J. (1994). Comprender la mente representacional. Paidós.

Raby, K. L., Roisman, G. I., Fraley, R. C., \& Simpson, J. A. (2015). The enduring predictive significance of early maternal sensitivity: Social and academic competence through age 32 years. Child Development, 86(3), 695-708. https://doi.org/10.1111/ cdev. 12325

Ramos, R., Yávar, V., Del Rio, A., Schettino, J., Bresciani, V., Gómez, D., Álvarez, C., \& Farkas, C. (2020). Tipo de parto y sensibilidad materna: Efectos en el desarrollo socioemocional de niños y niñas al año de 

el desarrollo emocional en la primera infancia. Revista de Sociología, 36(1), 68-82. https://doi.org/10.5354/0719-529X.2021.64427

edad. Acta Colombiana de Psicología, 23(2), 241-253. https://doi.org/10.14718/ACP. 2020.23.2.10

Reijntjes, A., Kamphuis, J. H., Prinzie, P., \& Telch, M. J. (2010). Peer victimization and internalizing problems in children: A meta-analysis of longitudinal studies. Child Abuse \& Neglect, 34(4), 244-252. https://doi.org/10.1016/j.chia bu.2009.07.009

Reiss, F. (2013). Socioeconomic inequalities and mental health problems in children and adolescents: A systematic review. Social Science \& Medicine, 90, 24-31. https://doi.org/10.1016/j.socscimed.2013.04.026

Rescorla, L. A., Achenbach, T. M., Ivanova, M. Y., Dumenci, L., Almqvist, F., Bilenberg, N., Bird, H., Broberg, A., Dobrean, A., Döpfner, M., Erol, N., Forns, M., Hannesdottir, H., Kanbayashi, Y., Lambert, M. C., Leung, P., Minaei, A., Mulatu, M. S., Novik, T. S., ... Verhulst, F. (2007). Epidemiological comparisons of problems and positive qualities reported by adolescents in 24 countries. Journal of Consulting and Clinical Psychology, 75(2), 351-358. http://doi.org/10.1037/0022-006X.75.2.351

Rescorla, L. A., Achenbach, T. M., Ivanova, M. Y., Harder, V. S., Otten, L., Bilenberg, N., Bjarnadottir, G., Capron, C., De Pauw, S. S. W., Dias, P., Dobrean, A., Döpfner, M., Duyme, M., Eapen, V., Erol, N., Esmaeili, E. M., Ezpeleta, L., Frigerio, A., Funget, D. S. S., ... Verhulst, F. (2011). International comparisons of behavioral and emotional problems in preschool children: Parents' reports from 24 societies.
Journal of Clinical Child \& Adolescent Psychology, 4O(3), 456-467. http://doi.org/ 10.1080/15374416.2011.563472

Rhoades, B. L., Warren, H. K., Domitrovich, C. E., \& Greenberg, M. T. (2011). Examining the link between preschool social-emotional competence and first grade academic achievement: The role of attention skills. Early Childhood Research Quarterly, 26(2), 182-191. https://doi.org/10.1016/j.ecresq.2010.07.003

Rodrigo, M. J., Máiquez, M. L., Martín, J. C., \& Byrne, S. (2008). Preservación familiar: Un enfoque positivo para la intervención con familias. Pirámide.

Ruffman, T., Slade, L., \& Crowe, E. (2002). The relation between children's and mother's mental state language and theory-of-mind understanding. Child Development, 73(3), 734-751. https://doi.org/10.1111/14678624.00435

Santelices, M. P., Farkas, C., \& Aracena, M. (2017). Evaluation of the effectiveness of a pilot program that promotes sensitive response in the educational staff of Chilean nursery schools. Children and Youth Services Review, 75, 87-95. https://doi.org/10.1016/j.childyouth. 2017.02.023

Santelices, M. P., Zapata, J., Fischersworring, M., Pérez, F., Mata, C., Barco, B., Olhaberry, M., \& Farkas, C. (2016). Intervenciones basadas en la mentalización para padres y educadores: Una revisión sistemática. Terapia Psicológica, 34(1), 71-80. http://doi.org/10.4067/S071848082016000100008 
Slaughter, V., \& Peterson, C. (2009). Maternal mental state talk and infants' early gestural communication. Journal of Child Language, 36(5), 1053-1074. https://doi.org/10.1017/S0305000908009306

Sroufe, L. A. (1996). Emotional development. The organization of emotional life in the early years. Cambridge University Press.

Strasser, K., \& Lissi, M. R. (2009). Home and instruction effects on emergent literacy in a sample of Chilean kindergarten children. Scientific Studies of Reading, 13(2), 175204. https://doi.org/10.1080/10888430902769525

Taumoepeau, M., \& Ruffman, T. (2008). Stepping stones to others' minds: Maternal talk relates to child mental state language and emotion understanding at 15, 24, and 33 months. Child Development, 79(2), 284-302. https://doi.org/10.1111/j.1467-8624.2007.01126.x

Trommsdorff, G., \& Cole, P. M. (2011). Emotion, self-regulation, and social behavior in cultural contexts. En X. Chen \& K. H. Rubin (Eds.), Socioemotional development in cultural context (pp. 131-163). Guilford Press.

Tsai, J. L., Louie, J. Y., Chen, E. E., \& Uchida, Y. (2007). Learning what feelings to desire: Socialization of ideal affect through children's storybooks. Personality and Social Psychology Bulletin, 33(1), 17-30. https://doi.org/10.1177/0146167206292749
Universidad de Chile, Facultad de Medicina, Escuela de Salud Pública Dr. Salvador Allende Gossen, Equipo Investigador Minoletti, A., Alvarado, R., Rayo, X., \& Minoletti, M. (2014). Sistema de salud mental de Chile [Segundo informe]. Organización Mundial de la Salud; Organización Panamericana de la Salud; Ministerio de Salud, Gobierno de Chile. https://www.who.int/mental_health/who_aims_ country_reports/who_aims_report_chile.pdf

Valdés, C., \& Errázuriz, P. (2012). Salud mental en Chile: El pariente pobre del sistema de salud. Clave de Políticas Públicas, (11), 1-10.

https://bit.ly/ValdesyErrazuriz2012

Werner, C., Linting, M., Vermeer, H., \& Van IJzendoorn, M. (2016). Do intervention programs in childcare promote the quality of caregiver-child interactions? A meta-analysis of randomized controlled trials. Prevention Science, 17(2), 259-273. https://doi.org/10.1007/s11121-015-0602-7

Widen, S. C., \& Russell, J. A. (2010). Young children's understanding of others' emotions. En M. Lewis, J. M. Haviland-Jones, \& L. Feldman (Eds.), Handbook of emotions (pp. 348-363). The Guilford Press.

Manuscrito recibido: 26-02-2021 Manuscrito aceptado: 09-07-2021 